\title{
Valor intrínseco y valor extrínseco en ética ambiental. Una alternativa antropocéntrica al instrumentalismo
}

\author{
Intrinsic and Extrinsic Value in Environmental Ethics. \\ An Anthropocentric Alternative to Instrumentalism
}

\author{
Juan Pablo Hernández Betancur*
}

Pontificia Universidad Javeriana, Colombia

\begin{abstract}
RESUMEN. En primer lugar, el artículo se propone especificar el debate entre no-antropocentristas y antropocentristas como uno entre quienes defienden que el ambiente tiene valor intrínseco, esto es, independiente de cualquier relación con lo humano; pero moralmente vinculante, y quienes lo niegan, esto es, afirman que solo tiene valor extrínseco. El artículo argumenta a favor de la segunda opción, pero defiende que esto no debe ser un obstáculo para desarrollar ciertas preocupaciones ambientalistas, pues el valor extrínseco no se reduce al instrumental. En relación a esto, se presenta el sentimentalismo como una alternativa según la cual hay formas de valoración de las cosas como fines en sí mismas y no solo como medios.
\end{abstract}

Palabras clave: Antropocentrismo; biocentrismo; ecocentrismo; argumento del último hombre; sentimentalismo.
Abstract. In the first place, I propose to specify the debate between non-anthropocentrists and anthropocentrists as one between those who defend that the environment has intrinsic value, that is, value that while independent of all relation to human beings is still morally binding, and those who deny this, i.e., assert that the environment only has extrinsic value. I argue for the second option but claim that this should not be an obstacle to address some of the worries of environmental ethics, since extrinsic value is not limited to instrumental value. In this regard, I present sentimentalism as an alternative according to which there are ways of valuing things as ends in themselves and not only as means.

Key words: Anthropocentrism; Biocentrism; Ecocentrism; the Last Man Argument; Sentimentalism.

\footnotetext{
*hernandez-juan@javeriana.edu.co / ORCID iD: https://orcid.org/0000-0001-8900-6852. 


\section{Introducción}

A pesar de ser una de las cuestiones centrales de la ética ambiental desde sus inicios y de ciertos altibajos en el nivel de atención recibida, el debate entre antropocentristas y no-antropocentristas sigue abierto. De manera general, la cuestión es si la ética ambiental debe estar centrada o no en el ser humano. Esto equivale a preguntar si la fuente de las demandas morales que conciernen al modo de conducirnos con respecto al ambiente o a ciertos conjuntos naturales (como veremos, el rango de objetos dependerá de la teoría) son otros seres humanos o si lo es el ambiente mismo ${ }^{1}$.

Antropocentrismo y no-antropocentrismo responden a un conjunto de preocupaciones, como la llamada crisis ecológica, que en muchos casos se comparten en una medida significativa. En esta medida, antropocentristas y no-antropocentristas con frecuencia están de acuerdo respecto de muchos juicios morales particulares, por ejemplo, que sería incorrecto talar un bosque que sea el hogar de una especie endémica para construir un complejo turístico privado. La diferencia entre los dos estriba en el tipo de razones que aducirían como fundamento de su juicio moral, diferencia que se retrae a desacuerdos de orden metafísico y metaético. En los años setenta y ochenta del siglo pasado, estas cuestiones se ventilaron especialmente en términos de teoría del valor; particularmente en relación a la pregunta de si el ambiente tiene valor intrínseco o no². El debate sobre el tipo de valor del ambiente perdió fuerza por unos años debido a posturas que sostenían que en últimas la cuestión práctica debía tomar precedencia sobre la metafísica y metaética, y que a nivel práctico podría no haber diferencia entre uno y otro bando del debate $^{3}$. Pero en años recientes, la cuestión ha suscitado un renovado interés entre filósofos ambientales ${ }^{4}$. Una de las motivaciones tras este cambio es la idea de que seguimos necesitando un buen marco teórico que permita capturar $-\mathrm{y}$ evaluar la justificación de- ciertas afirmaciones que ambientalistas en general (y no particularmente filósofos) quieren hacer sobre el ambiente y nuestra relación práctica con el mismo (moral y no moral) (McShane, 2007). Esta motivación es comprensible a la luz de las dificultades y frustraciones del debate práctico sobre casos concretos y políticas originadas en buena medida en las grandes diferencias entre los modos de valoración del ambiente de los distintos actores y las dificultades para comunicarlas y buscar formas de mediación entre las mismas. Adicional a esto, aportes recientes en metaética prometen poner a nuestra disposición formas de conceptualizar y explicar la cuestión del valor de formas que superen las limitaciones de las posturas de décadas anteriores ${ }^{5}$.

Por otra parte, en la literatura en español sobre ética ambiental, las discusiones sobre los fundamentos teóricos mismos del valor del ambiente, en contraste con propuestas que presuponen una solución específica a la cuestión, no son muy numerosas ${ }^{6}$. Esto es desafortunado justamente porque, por razones culturales e históricas, en la mayoría de los territorios hispanoparlantes las dificultades de conceptualización mencionadas en el párrafo anterior y sus repercusiones prácticas son especialmente acuciantes ${ }^{7}$. 
En este texto me propongo, por una parte, hacer una exposición general del problema del valor del ambiente en relación al debate entre antropocentristas y no-antropocentristas, y por otra, argumentar que, aunque debe descartarse la tesis de que el ambiente tiene valor intrínseco, ello no implica afirmar que solo tiene valor instrumental, pues hay otros tipos de valor extrínseco en términos de los cuales se podría dar curso a varias preocupaciones centrales de la ética ambiental.

En la siguiente sección repasaré algunas posturas no-antropocéntricas clásicas para mostrar el lugar que en ellas ocupa una teoría del valor. En la sección 3 propondré una especificación del debate entre antropocentristas y no-antropocentristas que evita confusiones habituales respecto de la teoría del valor. En la sección 4 expongo dos argumentos contra la tesis del valor intrínseco y en las secciones cinco y seis consideraré una posible réplica que se basa en un experimento mental muy influyente en ética ambiental. Esto permitirá mostrar la capacidad de ciertas explicaciones antropocéntricas para lidiar con el caso desde la afirmación de valores extrínsecos no-instrumentales.

\section{Del no-antropocentrismo a la teoría del valor}

De manera muy general, los no-antropocentristas sostienen que el ambiente, o entidades o todos que son partes del ambiente, tienen un tipo de valor independiente de su relación con los humanos pero que, sin embargo, nos impone demandas morales.

Paul Taylor, por ejemplo, afirma la existencia de este tipo de valor, que él llama inherente, con respecto a los seres vivientes. Al inicio de uno de sus textos más influyentes, afirma:

Además, e independientemente de, cualquiera de las obligaciones que podamos tener hacia nuestros prójimos humanos, también tenemos deberes hacia los seres salvajes vivientes por sí mismos (...) Nuestros deberes hacia las formas de vida no-humanas de la Tierra se basan en su estatus como entidades con valor inherente [inherent worth]. Tienen un tipo de valor que les pertenece en virtud de su propia naturaleza, y es este valor el que hace que sea incorrecto tratarlos como si existieran únicamente como meros medios para los fines humanos (Taylor, 1986, p. 13).

Holmes Rolston III, por su parte, refiriéndose al hecho de que la naturaleza produce constantemente variaciones como potenciales soluciones a problemas biológicos y ecosistémicos afirma:

[N]i la capacidad para sentir ni la consciencia son necesarios para que ocurran procesos inventivos. El hecho de que la naturaleza sistémica es inventiva es la raíz de todo valor, y todos los productos creados por la naturaleza tienen valor en la medida en que son logros inventivos (Rolston, 1988, p. 198).

Callicot ofrece una postura no-antropocéntrica más cualificada que contrasta fuertemente con la de Rolston:

Una cosa con valor intrínseco es [desde una perspectiva que tiene en cuenta el naturalismo científico] valiosa en razón 
de sí misma y por sí misma, pero no es valiosa en sí misma, es decir, con completa independencia de cualquier consciencia, pues, en principio, desde el punto de vista de la ciencia clásica normal ningún valor puede ser completamente independiente de una consciencia evaluadora. Defiendo que las especies no-humanas pueden poseer valor intrínseco en este sentido modificado (Callicott, 1989, pp. 133-134).

Pasajes como estos nos permiten apreciar tres puntos respecto de la teoría del valor en relación con la ética ambiental. Primero, la teoría pretende fundamentar deberes morales humanos. La clave para conseguirlo es atribuir un tipo de valor específico cuya posesión debe imponer demandas morales a los humanos. Segundo, el tipo de valor que se atribuye no es exactamente el mismo en todos los casos. Cada teoría supondrá una clasificación específica de tipos de valor y en muchos casos las clasificaciones no coinciden del todo, como veremos. Tercero, las entidades a las que se atribuye este tipo de valor tampoco son las mismas para todas las posturas. Para Taylor, como es evidente, los poseedores de este tipo de valor son los seres vivientes (biocentrismo), mientras que para Rolston III y Callicot, es todo el ambiente, incluyendo entidades orgánicas e inorgánicas (ecocentrismo) $)^{8}$.

Salta a la vista que esta última cuestión, el rango de atribución de valor, es secundaria con respecto a las dos primeras. Solo si existe cierto tipo de valor y este es capaz de fundamentar demandas morales, tiene sentido preguntarse qué entidades lo poseen en el contexto de la ética ambien- tal. En este texto, pues, me centraré en los dos primeros problemas.

\section{Tipos de valor}

Como he dicho, los tipos de valor intrínseco propuestos por filósofos ambientalistas no siempre son coincidentes, o cuando lo son, dicha coincidencia no es evidente. Por ello, a continuación propongo una taxonomía de los tipos de valor en la que trato de reunir y reorganizar algunas de las propuestas que se hallan en la literatura.

En un artículo muy influyente sobre teoría del valor, no sobre ética ambiental en particular, Christine Korsgaard distingue entre dos pares de oposiciones que tienden a ser fundidas. Por un lado, está la distinción entre valor como fin y valor como medio; y por otro la distinción entre valor extrínseco e intrínseco (Korsgaard, 1996). Empecemos por la última. Un objeto tiene valor intrínseco si su valor depende de propiedades intrínsecas, esto es, propiedades que el objeto posee independientemente de su relación con otras entidades. Quien desarrolló este concepto de manera explícita en el siglo XX fue G.E.M. Moore, y por ello a veces este concepto se ha llamado valor intrínseco mooreano ${ }^{9}$. De acuerdo a la definición de Moore, entonces, un objeto preserva su valor intrínseco, aunque sea lo único que exista ([1903] 1993).

Una consecuencia evidente de la noción de valor intrínseco es que es si algo lo tiene, su valor es final, esto es, no en tanto medio para un fin. Este último tipo de valor, el valor instrumental, es necesariamente extrínseco, es decir, emana de 
propiedades que dependen de la relación de dicho objeto con otros objetos. Aunque evidentemente hay propiedades relacionales e inclusive instrumentales en las que los humanos no somos ninguno de los términos de la relación, y estas son importantes en ética ambiental, en adelante me concentraré exclusivamente en relaciones con lo humano, y así entenderé los términos 'extrínseco' y 'relacional' ${ }^{10}$. Así pues, el valor instrumental es necesariamente extrínseco. Pero lo importante es que en la discusión sobre ética ambiental, con frecuencia se pasa por alto o al menos se subestima el hecho de que el valor instrumental no es el único tipo de valor extrínseco. Por ejemplo, hay gente que valora a Alejandro Magno porque admira su claridad de propósito, talento militar y liderazgo. Pero no es evidente que esta respuesta afectiva, la admiración de dicho personaje, sea la satisfacción o promoción de un deseo, preferencia o interés específico. Es más bien lo converso, la admiración, que implica una valoración no instrumental de Alejandro Magno, es fuente de intereses y preferencias específicas, como las de querer aprender más sobre la historia de Alejandro, querer que se preserve su memoria, etc. ${ }^{11}$. En este caso, el objeto es valorado como fin en sí mismo, pero la valoración depende de una respuesta humana específica, la admiración, y por ello el valor es extrínseco.

Las aclaraciones anteriores permiten apreciar dos formas en que puede interpretarse la oposición entre antropocentrismo y no-antropocentrismo. O bien la disputa es acerca de si las entidades naturales tienen valor intrínseco o no, o bien es acerca de si tienen valor extrínseco no-ins- trumental, esto es, como fines en sí mismos. Una respuesta positiva a la primera forma de plantear la cuestión, es decir, la afirmación de la existencia de valor intrínseco en la naturaleza, define una postura no-antropocentrista con hondos compromisos metafísicos, como veremos. Una de sus consecuencias es que las entidades naturales poseen valor con total independencia de cómo respondamos los humanos (o cualquier agente moral posible) al mismo, y más aún, aunque no existiéramos. Como he señalado, esta es una postura muy reconocida en ética ambiental y de hecho está en el origen mismo uno de los programas más influyentes desde sus inicios, a saber, la ecología profunda.

Pero se puede responder negativamente a la primera pregunta y positivamente a la segunda. El caso de Callicot es ilustrativo. En el pasaje citado al inicio, Callicot niega que tenga sentido atribuir valor independiente de las evaluaciones humanas, por lo tanto, el valor atribuido, que él llama "intrínseco en sentido modificado", en ningún caso puede ser intrínseco en el sentido que en este trabajo hemos asumido, es decir, mooreano, sino extrínseco. Sin embargo, Callicot cree que el valor atribuido no necesariamente es instrumental. En virtud de nuestras disposiciones podemos valorar no instrumentalmente las entidades naturales. En este sentido, Callicot se autodenomina un noantropocentrista, más específicamente, un ecocentrista (Callicott, 1984). Esta etiqueta es la misma bajo la que se sitúa Rolston, a pesar de que, a diferencia de Callicot, para él el ambiente sí tiene valor intrínseco mooreano. En lo que sigue, reservaré el término 'no-antropocentrismo' 
para posturas como la de Rolston, esto es, para las que afirman la existencia de valor intrínseco mooreano. Propiamente son estos quienes defienden que los deberes morales con el ambiente se fundamentan en algo independiente de nosotros los seres humanos $^{12}$. En la siguiente sección quiero considerar los prospectos del no-antropocentrismo, entendido en este sentido fuerte. Para hacer esto será necesario empezar por abordar la cuestión de cómo la atribución de valores puede fundamentar deberes morales.

\section{Valor intrínseco y razones para actuar}

En el contexto de la filosofía moral y de la ética ambiental, la atribución de valor en general está necesariamente ligada a la cuestión normativa de cómo debemos actuar nosotros los humanos (o cualquier agente moral), por lo tanto, la noción de valor debe ser normativamente activa. Esta es una preocupación importante para Taylor, por ejemplo, uno de los defensores de la noción de valor intrínseco. Tras afirmar, como vimos, que este valor es independiente de evaluaciones humanas, escribe:

La afirmación de que una entidad tiene valor inherente ${ }^{13}$ debe ser entendida aquí como implicando dos juicios morales: (1) que la entidad es merecedora de preocupación y consideración moral, o, en otras palabras, que debe ser considerada como un sujeto moral, y (2) que todos los agentes morales tienen, prima facie, el deber de promover o preservar el bien de la entidad como un fin en sí y en virtud de la entidad de la que es un bien (Taylor, 1986, p. 75).
¿Es sostenible este tipo de posición? Los prospectos en mi opinión, no son buenos. Para mostrar esto, no examinaré argumentos particulares a favor de la tesis del valor intrínseco, como los ofrecidos por Taylor mismo ${ }^{14}$, sino que me concentraré en esta sección en una crítica general a la idea misma de valor intrínseco. Esta crítica deriva de discusiones importantes en metaética y filosofía de la acción.

Como he dicho, la idea de valor intrínseco recibió fuertes críticas desde que fue articulada explícitamente por Moore en sus Principia Ethica en el contexto de la filosofía moral general. Una de las expresiones más influyentes de esta crítica para discusiones recientes es la propuesta por Mackie bajo el llamado argumento de la rareza. Refiriéndose al valor intrínseco como valor moral objetivo, Mackie afirma que este tipo de valor supuestamente

le proporciona a quien lo conoce tanto una dirección como un motivo que supera a cualquier otro; que algo sea bueno le dice a quien lo conoce que lo persiga y que lo haga su propósito. Un bien objetivo sería buscado por cualquiera que lo conociera, no debido al hecho contingente de que esta persona, o toda persona, está constituida de tal modo que desea dicho fin, sino simplemente porque tiene incorporada la índole de ser perseguida. (Mackie, 1990, p. 40).

En otras palabras, el tipo de valor en discusión debe ser intrínseco sin dejar de ser normativa y motivacionalmente activo. Debe ser independiente de las respuestas motivacionales humanas y a la vez ser capaz de proporcionar una razón y una moti- 
vación a los agentes humanos. Para que algo así sea posible, razona Mackie de manera algo sarcástica en la última línea citada, lo normativo y lo motivacional no deberían ser propiedades relacionales sino pertenecer al objeto mismo. Si concedemos esto a Mackie, parece difícil escapar a su conclusión de que dichas propiedades serían muy diferentes a las propiedades de las cuales tenemos conocimiento en la naturaleza, serían extremadamente extrañas. Para Mackie, la consecuencia es que la teoría de que existen valores intrínsecos es falsa.

Es interesante anotar que el argumento de Mackie ha recibido una crítica importante por parte de John McDowell y otros. Ellos arguyen que, al concebir el valor moral como una cualidad primaria (en términos de Locke), Mackie pasa por alto la posibilidad de que sea "objetivo" en el sentido de estar "ahí afuera", aunque dependa de la sensibilidad humana, a la manera de las cualidades secundarias (McDowell, 1998a, 1998b; Prinz, 2007). Sin embargo, lo más significativo es que esta crítica deja intacto el poder del argumento de Mackie en contra de una concepción de los valores como cualidades primarias, y esta es justamente la noción de valor intrínseco que nos interesa en el momento. Para una concepción como esta, McDowell mismo es tan o más radical que Mackie y sugiere que el cargo que debería levantarse contra el defensor de una noción tal de valor debería ser el de incoherencia y no, simplemente, el de falsedad (McDowell, 1998a). Así es como pone el punto en un pasaje particularmente incisivo:

$[\mathrm{P}]$ arece imposible -al menos una vez se reflexiona sobre esto- tomarse en serio la idea de algo que es como una cualidad primaria en tanto simplemente está ahí, independientemente de la sensibilidad humana, pero que es, sin embargo, intrínsecamente (no en cuanto condicionada por las contingencias de la sensibilidad humana) capaz de producir una "actitud" o estado de la voluntad en quien llega a percibirla (McDowell, 1998b, p. 132).

La imposibilidad de valores intrínsecos se hace más nítida si tomamos un decurso por la teoría de la acción y reflexionamos sobre lo que es necesario para que haya poder normativo y motivacional. En su clásico artículo 'Razones internas y externas', Bernard Williams (1981) se preguntaba si podía atribuirse a un agente una razón para actuar que fuera independiente de su aparato motivacional, es decir, en sus términos, una 'razón externa'. La pregunta es central para nuestros propósitos, pues, como hemos visto, atribuir valor intrínseco en ética ambiental debe tener, por una parte, consecuencias normativas, y por otra, estas consecuencias deben ser independientes de las contingencias psicológicas humanas, entre las que naturalmente entra (o quizás son lo mismo), el aparato motivacional del agente $^{15}$. Por lo tanto, la existencia de valor intrínseco implica la existencia de razones externas. Si no existen razones externas, entonces no existe valor intrínseco.

Pues bien, la respuesta de Williams a la pregunta por la existencia de razones externas es negativa. Su argumento parte del punto generalmente aceptado de que la atribución de razones tiene una doble función. Por una parte, busca exhibir la justificación que tiene o tendría el agente para 
actuar de cierta manera. Aquí está en plena luz el carácter normativo de las razones. Por otra parte, la atribución de razones busca también explicar las acciones del agente. La existencia de razones debe dar cuenta de la producción de la acción. El problema es, para Williams, que estas dos funciones realmente no pueden separarse de manera tajante. La idea de tener razones para actuar debe servir, a una vez, para explicar y para justificar. Quien defiende la existencia de razones externas se concentra en la primera función, pero el precio es que pierde todo recurso para cumplir con la segunda, pues no es posible explicar la acción sin apelar a las disposiciones motivacionales del agente:

[N]ada puede explicar la acción (intencional) de un agente excepto algo que lo motive a actuar de esa forma. Así que además de la verdad de cierto enunciado sobre una razón externa, es necesario algo adicional para explicar la acción, alguna conexión psicológica (p. 107).

Si el argumento de Williams es sólido, entonces el defensor de la existencia de valor intrínseco está en serios aprietos, pues no puede independizar la noción de valor de las contingencias humanas y a la vez querer preservar su potencia normativa y motivacional ${ }^{16}$.

A mi juicio, los argumentos anteriores muestran que la noción de valor intrínseco es indefendible. Sin embargo, un defensor del valor intrínseco del ambiente podría replicar al anterior curso argumentativo invocando una figura central de la ética ambiental, el "último hombre" o la "última persona" (en formulaciones más recientes). Veamos.

\section{El argumento de la última persona y el valor intrínseco}

A comienzos de los años setenta, Richard Sylvan propuso un experimento mental con el fin de inducir la intuición de que el ambiente tiene valor intrínseco. Desde entonces, esta propuesta ha tenido una influencia masiva sobre la ética ambiental (Routley, 1973 ${ }^{17}$. El núcleo del experimento mental se puede plantear así: ¿sería incorrecto que la última persona sobre la Tierra, justo antes de morir, destruyera (de manera indolora) todo ser vivo? ${ }^{18}$ Para muchos, la respuesta es claramente positiva: la acción destructiva es incorrecta. En lo que sigue, no pondré en duda esta intuición, aunque ciertamente está lejos de ser universalmente compartida. La supuesta lección del experimento es que, dado que destruir la vida en la Tierra no tendría efectos sobre los seres humanos, entonces la razón por la que la acción destructiva es errónea debe ser no-antropocéntrica. Así pues, el argumento de la última persona parece mostrar que hay valor intrínseco en el ambiente. La fuerza de esta intuición podría entonces hacer contrapeso a las objeciones planteadas anteriormente contra la tesis del valor intrínseco.

El argumento, sin embargo, está muy lejos de ser exitoso. El problema central es que el escenario propuesto no permite realmente demostrar que el juicio de condena sobre la última persona sea independiente de nuestra sensibilidad. Quizás se puede admitir que el escenario está diseñado de modo que descarta dos cosas: uno, que nuestras respuestas al mismo sean respuestas al efecto previsto sobre otros seres humanos; dos, que nuestras respuestas deriven de consideraciones instrumentales. 
En el escenario no hay otros seres humanos que puedan verse afectados y el ambiente ha sido despojado de todo valor instrumental. Pero el escenario no excluye de ninguna manera que nuestra reacción siga siendo "antropocéntrica", dependiente de nuestras respuestas y aparato evaluativo contingente. La prueba de esto es que nuestra reacción de condena moral puede ser explicada apelando a valores extrínsecos no-instrumentales.

\section{Valor extrínseco y la última persona}

En lo que queda de este artículo voy a considerar cierta vía para explicar y dar un marco teórico tanto al problema de la última persona como a la ética ambiental en general que evita tanto apelar al valor intrínseco como a reducir todo valor al tipo instrumental. Empiezo por consideraciones relativamente libres de compromisos teóricos fuertes y luego esbozo un marco más robusto y comento su potencial.

\section{Valor estético, simbólico, cultural y sus parientes}

La existencia de valores estéticos, simbólicos, históricos, culturales y otros de este tipo, ha sido reconocida por no-antropocentristas desde hace tiempo, pero típicamente es desestimada de manera expedita como un buen recurso para la ética ambiental (Rolston, 1988; Taylor, 1986). Por otra parte, desde el bando antropocentrista ha habido varios intentos de mostrar la importancia de estos tipos de valor, especialmente el estético (Carter, 2010; Tafalla, 2005) ${ }^{19}$. Creo, con los antropocentristas, que este tipo de valor no debe desestimarse, y una buena prueba de ello es que apelar a este permite tratar con el problema de la última persona. Por supuesto, estos son distintos tipos de valor y una discusión detallada debería considerarlos por separado. Sin embargo, creo que, en una aproximación inicial y general, como la que intento, pueden tratarse todos en conjunto sin perder justeza.

Parece difícil negar que estos valores existen y es altamente plausible que a veces se acoplen al ambiente o a ciertas entidades naturales. Desde este tipo de perspectiva, la última persona actúa mal porque destruye algo considerado por nosotros como bello, por ejemplo, o que tiene cierto significado para nosotros. Este "nosotros" refiere a los evaluadores del escenario, quienes (hemos supuesto) compartimos la intuición de que el acto es malo. Desde este punto de vista, el objeto directo de nuestra reacción moral es el efecto sobre el ambiente (no la última persona como tal), pero ello no depende de que le atribuyamos valor intrínseco al mismo.

Una reacción más o menos natural a esta posibilidad es protestar diciendo que no tiene sentido que evaluemos la acción desde nuestra sensibilidad estética, simbólica, histórica, etc., ya que la acción misma, de darse, no tendría efecto real sobre nosotros. Por estipulación, nosotros no estaríamos presentes en el escenario. La réplica diría, por ejemplo, que no tiene sentido considerar que el ambiente posee belleza en unas circunstancias en que no va a haber nadie que lo considere bello. Esto, a mi juicio, equivale o está muy cerca de equivaler a negar que el valor que está en juego sea realmente no-instrumental, pues parece suponer que la razón por la que atribuimos valor estético es que el objeto cumpla (o pueda 
cumplir) ${ }^{20}$ alguna función respecto de nosotros (u otras posibles personas). En el caso del valor estético, una función plausible sería la de producir cierto tipo de experiencias; en el caso del valor simbólico, podría ser la de producir cierto tipo de reacciones emocionales y motivacionales; y así con los demás casos. Si la razón de la atribución de valor fuera la función del objeto, entonces el valor sería instrumental. Así, pues, si alguien concibe las obras de arte estrictamente como objetos de consumo, entonces puede asimilar el valor estético a un tipo de valor instrumental. Sin consumidor (de la obra), no habrá valor.

Pero la razón de la atribución de valor no necesariamente es la función que puede cumplir el objeto. Bien puede ser que la razón por la que atribuimos valor estético es simplemente que el objeto tiene ciertas características que percibimos. Por ello, decir que $\sin$ (potencial) consumidor o beneficiario de una función no habrá valor es mucho más restrictivo que decir que sin evaluador no habrá valor, que es lo que exige el valor extrínseco no-instrumental. Puede que el valor estético de algo dependa de nuestras evaluaciones, pero de aquí no se sigue que la existencia de ese valor dependa de que podamos disfrutarlo o consumirlo ${ }^{21}$.

\section{Sentimentalismo}

Las observaciones anteriores tienen el carácter de mostrar un fenómeno común, la atribución de valor estético, cultural, etc., y revelar un punto importante para la teoría del valor, a saber, que este valor no es instrumental ni intrínseco. A continuación, quisiera mostrar que el punto anterior puede ponerse en un contexto teórico más amplio y robusto, que es prometedor para una ética ambiental, el de la teoría metaética sentimentalista.

El sentimentalismo moral, desarrollado por los empiristas ingleses del siglo XVIII, es una postura metaética ambiciosa en tanto ofrece una teoría sobre la naturaleza de los juicios y las apreciaciones morales en general. Los sentimentalistas sostienen que las evaluaciones morales dependen de nuestras disposiciones emocionales. Por poner un ejemplo, el juicio de que cierto acto es una injusticia es verdadero en la medida en que se conecte con una emoción de indignación. Es la indignación la que hace al acto injusto.

El sentimentalismo ha vivido un resurgimiento en las últimas décadas en el contexto de la filosofía moral y la metaética en general $^{22}$. En el contexto de la ética ambiental, su exponente más conocido es Ca1 licot ${ }^{23}$, aunque para sus formulaciones más importantes, anteriores al resurgimiento del sentimentalismo en filosofía moral, se basó en el sentimentalismo clásico. Más recientemente, los nuevos trabajos sentimentalistas han empezado a ser explorados con gran interés por otros filósofos ambientales ${ }^{24}$.

Debe ser ya evidente el aporte que puede hacer el sentimentalismo contemporáneo a la ética ambiental. Si se acepta esta teoría, entonces la posibilidad de explicar el caso de la última persona está al alcance de la mano. Dado que para el sentimentalista nuestros juicios morales dependen de nuestras disposiciones emocionales, basta postular que quienes condenamos a la última persona tenemos una disposición emocional moral asociada a la preservación y protección del ambiente. Para poner esta idea en el contexto más amplio de la teoría del valor, algunas de nuestras disposiciones emocionales consti- 
tuyen, como ha argüido Katie McShane (2007, 2011), actitudes de valoración noinstrumental. Cuando amamos o cuando admiramos tomamos el objeto de nuestra reacción como un fin en sí mismo ${ }^{25}$. Estas actitudes emocionales generan, entonces, valor extrínseco no-instrumental.

Vale la pena llamar la atención sobre el hecho de que las actitudes de valoración no-instrumental dan lugar tanto a valores no morales como a otros claramente morales. La admiración, por ejemplo, no necesariamente fundamenta deberes morales. Que debido a mi admiración por Alejandro Magno yo quiera y considere mi deber ayudar a preservar su memoria no necesariamente constituye una cuestión moral. Lo mismo ocurre, en principio, con las valoraciones estéticas. Pero por otra parte, la indignación parece involucrar la dimensión moral de forma inmediata. El daño causado a ese tercero que valoramos por sí mismo y no instrumentalmente es para nosotros una injusticia. El sentimentalismo, entonces, podría explicar y dar un marco teórico, por una parte, a las valoraciones no-instrumentales no morales con respecto al ambiente, como las mencionadas al comienzo de esta sección (valores estéticos, simbólicos, etc.), y por otra, a las valoraciones no-instrumentales directamente morales. Estas últimas, por supuesto, son mucho más robustas y vinculantes (para quienes tienen las disposiciones emocionales pertinentes), lo cual podría dar razón de la importancia de los deberes ambientales para muchos ambientalistas y para ciertas comunidades.

Una fuente de discusión importante para el sentimentalismo contemporáneo es que en principio consiste en una teoría puramente descriptiva (tales y cuales actitudes morales dependen de tales o cuales disposiciones emocionales) y carece por tanto de fuerza normativa: del hecho de que alguien tenga la disposición emocional que le permite percibir ciertas demandas morales con respecto del ambiente, no se sigue que otra persona sin dichas disposiciones tenga las obligaciones morales correspondientes ${ }^{26}$. Sin embargo, termino anotando que, en el peor de los casos, aunque el sentimentalismo no permita justificar que los seres humanos debamos tener las disposiciones emocionales de las cuales dependería una ética ambiental, sí proporciona elementos para pensar qué sería necesario para que quienes no las tengan empiecen a ver o ser sensibles a demandas morales concernientes al ambiente, esto es, qué tipo de disposiciones podrían inculcarse y cultivarse. Para ello, por una parte, resulta fundamental una labor que muestre el tipo de disposiciones que impiden ver el ambiente como fuente de demandas morales. Una enseñanza central de análisis como los ofrecidos desde el ecofeminismo, por ejemplo, muestran estas disposiciones bajo una luz crítica que las vincula con formas de dominación e injusticia mucho más amplias, lo cual las hace indeseables ética, políticamente y quizás incluso existencialmente $^{27}$. Por otra parte, el sentimentalismo sugiere que la formación de nuevas disposiciones no tiene que ser promovida como la conclusión de ciertos argumentos. Como muchos han aceptado de tiempo atrás, la argumentación no es la única manera razonable, o al menos no irracional, de cambiar las opiniones y modos de ver de las personas. Como sostiene McDowell, cuando se trata de formar un ser humano en una cultura, la argumentación es, en el mejor de los casos un recurso ocasional (McDowell, 1998b) ${ }^{28}$. 


\section{Bibliografía}

Arias Maldonado, M. (2011). Hacia un constructivismo realista: de la naturaleza al medio ambiente. Isegoría: Revista de Filosofia Moral Y Política, (44), 285-301.

Bradley, B. (2006). Two Concepts of Intrinsic Value. Ethical Theory and Moral Practice, 9(2), 111-130.

Callicott, J. B. (1984). Non-antropocentric Value Theory and Environmental Ethics. American Philosophical Quartely, 21(4), 299-309.

Callicott, J. B. (1989). In Defense of the Land Ethic. Albania: SUNY Press.

Carter, A. (2010). Biodiversity and All That Jazz. Philosophy and Phenomenological Research, 80(1), 58-75.

D’Arms, J., \& Jacobson, D. (2000). Sentiment and Value. Ethics, 110(4), 722-748.

Frankfurt, H. (2004). The Reasons of Love. Princeton: Princeton University Press.

Frankfurt, H. (2007). Necesidad, volición y amor. Madrid: Katz.

Gilligan, C. (1982). In a Different Voice: Psychological Theory and Women's Development. Cambridge, Mass; London: Harvard University Press.

Gruen, L. (1997). Revaluing Nature. In K. J. Warren (Ed.), Ecofeminism: Women, Culture, Nature (pp. 356-374). Bloomington: Indiana University Press.

Gudynas, E. (2010). La senda biocéntrica: valores intrínsecos, derechos de la naturaleza y justicia ecológica. Tabula Rasa, (13), 45-71.

Herguedas, F. A. (2006). Del valor intrínseco de la naturaleza. Isegoría: Revista de Filosofia Moral Y Política, (34), 261-275.

Kasperbauer, T. J. (2015). Naturalizing Sentimentalism for Environmental Ethics. Environmental Ethics, 37(2).
Korsgaard, C. M. (1996). Two Distinctions in Goodness. In Creating the Kingdom of Ends (pp. 249-274). Cambridge: Cambridge University Press.

Lo, Y. S. (2006). Making and Finding Values in Nature: From a Humean Point of View. Inquiry, 49(2), 123-147.

Mackie, J. L. (1990). Ethics: Inventing Right and Wrong. London and New York: Penguin Books Limited.

McDowell, J. (1998a). Aesthetic Value, Objectivity, and the Fabric of the World. In Mind, value and reality (pp. 112-130). Cambridge, Mass: Harvard University Press.

McDowell, J. (1998b). Might there be external reasons? In Mind, value and reality (pp. 95-111). Cambridge, Mass: Harvard University Press.

McDowell, J. (1998c). Values and Secondary Qualities. In Mind, value, and reality (pp. 131-150). Cambridge, Mass: Harvard University Press.

McShane, K. (2007). Why environmental ethics shouldn't give up on intrinsic value. Environmental Ethics, 29(1), 43-61.

McShane, K. (2011). Neosentimentalism and Environmental Ethics. Environmental Ethics, 33(1), 5-23.

Moore, G. E. (1993). Principia Ethica. Revised Edition. (T. Baldwin, Ed.). Cambridge: Cambridge University Press.

Neira Brito, F. (2006). Representaciones de la naturaleza en la Amazonía ecuatoriana: ¿subsistencia local o conservación global? Iconos. Revista de Ciencias Sociales, (25), 57-65.

Nichols, S. (2004). Sentimental Rules. Oxford: Oxford University Press.

Norton, B. G. (1984). Environmental Ethics and Weak Anthropocentrism. Environmental Ethics, 6(2), 131-148. 
Norton, B. G. (1991). Toward Unity Among Environmentalists. Nueva York: Oxford University Press.

O'Neill, J. (1992). The Varieties of Intrinsic Value. Monist, 75(2), 119-137.

Peterson, M., \& Sandin, P. (2013). The Last Man Argument Revisited. Journal of Value Inquiry, 47(1), 121-133.

Pigrau Solé, A. (Ed.). (2013). Pueblos indígenas, diversidad cultural y justicia ambiental. Un estudio de las nuevas constituciones de Ecuador y Bolivia. Valencia: Tirant lo Blanch.

Plumwood, V. (1997). Androcentrism and Anthropocentrism: Parallels and Politics. In K. J. Warren (Ed.), Ecofeminism: Women, Culture, Nature (pp. 327-355). Bloomington, Indianapolis: Indiana University Press.

Prinz, J. J. (2007). The Emotional Construction of Morals. Cambridge: Cambridge University Press.

Robertson, J. (2001). Internalism, Practical Reason, and Motivation. In E. Millgram (Ed.), Varieties of Practical Reasoning (pp.
127-151). Cambridge, Mass: Oxford University Press.

Rolston, H. (1988). Environmental Ethics. Philadephia: Temple University Press.

Routley, R. (1973). Is There a Need for Environmental Ethics? Proceedings of the XVth World Congress of Philosophy, 205-210.

Singer, P. (2011). Practical Ethics. London and Stanford: Cambridge University Press. Slote, M. (2010). Moral Sentimentalism. Oxford: Oxford University Press.

Tafalla, M. (2005). Por una estética de la naturaleza: la belleza natural como argumento ecologista. Isegoría: Revista de Filosofía Moral Y Política, (32), 215-226.

Taylor, P. (1986). Respect for Nature. Princeton: Princeton University Press.

Weber, Z. (2017). Intrinsic Value and the Last Last Man. Ratio, 30(2), 165-180.

Williams, B. (1981). Internal and external reasons. In Moral Luck. Philosophical Papers 1973-1980. Cambridge: Cambridge University Press.
${ }^{1}$ A lo largo de este texto utilizaré el término "ambiente" para referirme de manera relativamente flexible a lo que con frecuencia se llama "la naturaleza". Escojo el término por considerarlo más neutro respecto de varias cuestiones importantes en ética ambiental, como la dicotomía entre naturaleza y cultura, y la noción de lo salvaje, las cuales no abordaré.

${ }^{2}$ Algunos textos clásicos de este debate son Callicott, 1984; Rolston, 1988; O’Neill, 1992; Routley, 1973 y Taylor, 1986.

${ }^{3}$ Norton es uno de los más conocidos e influyentes defensores de esta idea de la convergencia práctica entre antropocentristas y no-antropocentristas (1984, 1991).

${ }^{4}$ Por ejemplo, Lo, 2006; McShane, 2007, 2011; Peterson \& Sandin, 2013; Weber, 2017.
${ }^{5}$ Esta motivación se puede encontrar en Kasperbauer, 2015; Lo, 2006; McShane, 2011, a pesar de las diferencias entre los mismos. Este es un punto que elaboraré más adelante.

${ }^{6}$ Pero véase: Arias Maldonado, 2011; Herguedas, 2006; Tafalla, 2005.

${ }^{7}$ En el contexto latinoamericano, por ejemplo, es frecuente constatar grandísimas diferencias éticas y prácticas entre los modos de valoración del ambiente de culturas campesinas e indígenas, por una parte, y corporaciones y Estados, por otra. Para un panorama y reflexión sobre este punto, véase Gudynas, 2010; Neira Brito, 2006; y los estudios en Pigrau Solé, 2013.

${ }^{8}$ Otra posición bien conocida a este respecto es el sensocentrismo, según la cual los seres sintientes poseen valor intrínseco (por ejemplo, Singer, 2011). Cabe 
anotar, sin embargo, que la discusión sobre valor intrínseco se ha dado especialmente en relación al biocentrismo y el ecocentrismo, probablemente porque estas son posturas más fuertes y por ende más difíciles de defender a este respecto, lo cual no significa que el sensocentrismo no sea una postura aún polémica.

${ }^{9}$ En Bradley, 2006, quien discute distintas formulaciones de este tipo de valor y los principios que implica. En ética ambiental, esta distinción aparece, por ejemplo, en O’Neill, 1992; Taylor, 1986; (como 'inherent worth'), Rolston III, 1988; y Peterson y Sandin, 2013; entre muchos otros.

${ }^{10}$ Sobre esta idea de valor instrumental no dependiente de lo humano, ver O’Neill, 1992; Taylor, 1986.

${ }^{11}$ Un caso más contundente es el del amor, que requeriría una reflexión detallada que no puedo ofrecer aquí. Para una discusión iluminadora sobre la relación entre amor y valoración, véase Frankfurt, 2004 y 2007.

${ }^{12}$ En la medida en que se tengan claras las distinciones anteriores, la cuestión de cómo aplicar el término 'valor intrínseco' es estrictamente terminológica y por ende estipulativa. McShane (2007), por ejemplo, decide llamar 'valor intrínseco' a lo que yo llamo 'valor extrínseco no-instrumental'.

${ }^{13}$ Para los propósitos de este escrito, traduzco 'inherent worth 'por 'valor inherente', que no debe confundirse ni con 'intrinsic worth' ni con 'intrinsic value', empleadas por Taylor con otros significados. En boca de Taylor, el valor inherente es el que aquí llamo intrínseco.

${ }^{14}$ Cabe anotar, sin embargo, que Taylor es consciente de que su argumentación no es deductiva y tiene un potencial persuasivo limitado. Esto es reflejo, en mi opinión, de que, como toda defensa de la existencia de valor intrínseco, enfrenta las dificultades que voy a examinar.

${ }^{15}$ Quizás son lo mismo, si se tiene una concepción amplia del aparato motivacional, una que incluya disposiciones emocionales, convicciones, etc., y no solo deseos. Esta concepción amplia es, de hecho, la de Williams mismo en el artículo mencionado.

${ }^{16}$ Para una presentación iluminadora del debate abierto por Williams, ver Robertson, 2001.

${ }^{17}$ Routley cambió su apellido a Sylvan en 1983. El uso común, sin embargo, es citar este trabajo clásico por el apellido que tenía su autor en el momento de aparición del artículo.

${ }^{18}$ Hay varias versiones del experimento mental. Ver, por ejemplo, Weber 2017.

${ }^{19}$ En términos del rango de atribución de valor intrínseco, Tafalla es sensocentrista. Sin embargo, su argumento a favor del valor del ambiente se basa en respuestas humanas (estéticas) y no de todos los seres sintientes, lo que significa que en lo que toca a la teoría del valor del ambiente sería antropocentrista. Agradezco a un evaluador anónimo por conducirme a aclarar este punto.

${ }^{20}$ En los paréntesis registro la relevancia de un análisis contrafáctico para estos casos, pero no creo necesario llamar la atención más sobre este punto.

${ }^{21}$ Para otra forma de plantear este punto de que el valor estético es no instrumental, ver Tafalla, 2005.

${ }^{22}$ McDowell, 1998a, 1998b; Nichols, 2004; Prinz, 2007; Slote, 2010. Vale la pena señalar la relación entre el sentimentalismo contemporáneo y la ética del cuidado. Aunque esta última típicamente no se presenta como una teoría metaética, ofrece recursos importantes que pueden ser empleados por el sentimentalismo. La teoría de Slote, por ejemplo, está fuertemente influenciada por la ética del cuidado de Gilligan (1982).

${ }^{23}$ Por ejemplo en 1984, 1989.

${ }^{24}$ Kasperbauer, 2015; McShane, 2007, 2011.

${ }^{25}$ McShane de hecho habla de 'intrinsic valuing attitudes' vs. 'extrinsic valuing attitudes'. Como dije antes, yo he reservado el término 'intrínseco' para valor mooreano, mientras McShane se permite emplearlo en relación a lo que es valioso por sí mismo y no por otra cosa.

${ }^{26}$ Este es justamente el corazón del debate entre neosentimentalistas y lo que podríamos llamar sentimentalistas sin más. Un planteamiento muy importante del neo-sentimentalismo se encuentra en McDowell, 1998a, $1998 \mathrm{c}$, más recientemente ha sido elaborado en D'Arms y Jacobson, 2000, y en el contexto de la ética ambiental es central en McShane, 2011. Una defensa del sentimentalismo sin más contra el neo-sentimentalismo en ética ambiental, se encuentra en Kasperbauer, 2015.

${ }^{27}$ Ver por ejemplo Gruen, 1997 y Plumwood, 1997. Aunque el foco del ecofeminismo no ha sido la teoría del valor, propuestas como las referidas, hacen explícito de manera muy aguda, aunque en otros términos, el vínculo entre esta postura y lo que yo aquí he llamado valor antropocéntrico no instrumental.

${ }^{28}$ Este artículo se escribió como parte del proyecto 6423 de la Facultad de Filosofía y el Instituto de Bioética de la Pontificia Universidad Javeriana. Agradezco a José Edwin Cuéllar y a los demás integrantes del grupo por las invaluables discusiones y por su apoyo.También les agradezco a los evaluadores anónimos, sus valiosas sugerencias para mejorar este trabajo. 